>> Fernando Barbosa ${ }^{1}$

fernandobarbosa.reorient@gmail.com

\section{China: Entre el sueño y la utopía}

\section{China: Between the dream and the utopia}

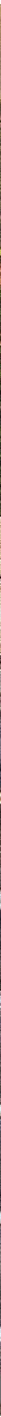

Fernando Barbosa, Politólogo de la Universidad de los Andes. Desde 1973 se ha dedicado a los temas de Asia y, particularmente, de Japón: en el sector privado y en el público, la diplomacia, la academia, el periodismo, la consultoría y la traducción. 
He is your friend, let him dream

Jack Kerouac

\section{Resumen}

El propósito de este artículo es el de plantear que el formidable crecimiento de China en las últimas décadas se ha inspirado en el denominado "sueño chino". Se mostrará que este propósito corresponde a un proceso de gran aliento que tiene raíces profundas y que está anclado en la historia y en la cultura de los chinos.

Con base en literatura académica reciente y relevante que aporta nuevas miradas y que plantea nuevos interrogantes se busca llamar la atención de los investigadores para enriquecer el debate sobre lo que significa China para el mundo en este momento. En tal sentido, lo que se busca plantear es la apertura de un abanico de dudas y de preguntas que permitan avanzar en el entendimiento de esta realidad.

\section{Palabras clave}

China, sueño chino, armonía, el cinturón y la ruta, CYI.

\section{Abstract}

The purpose of this essay is to advocate that the formidable economic growth that China has experienced in the most recent decades is both inspired and established upon the foundation of the "Chinese dream". This purpose corresponds to a process of great encouragement that has deep roots and is anchored in the history and Chinese culture.

Based on recent and relevant academic literature that provides new perspectives and that raises new enquiries, it seeks to draw the attention of researchers to enrich the debate on what China means for the world at this time. In this sense, what is sought to be raised is the opening of a range of doubts and questions that allow us to advance in the understanding of this reality.

\section{Keywords}

China, Chinese dream, harmony, belt and road, BRI.

\section{Introducción}

El soft power es la nueva denominación para hablar de la seducción de que se han valido los poderes en el curso de la historia para atraer a los de afuera y consolidarse interna y externamente. En el siglo XIX, la potencia dominante, Inglaterra, usó tanto su poder militar como su poder mercantil y naval para ejercer su dominio. Francia, que no competía al mismo nivel, nos embelesó con un refinado tipo de imperialismo (Shawcross, 2018) en el que el gusto fue el baluarte: se empezaba por el idioma, la literatura, los vinos, los perfumes, la moda. Hubo intentos mayores como los que emprendió Napoleón, o el de Maximiliano en México, en los que tampoco tuvo éxito si bien terminó transformándose en algo que le abrió más espacios que los que posiblemente hubiera podido realizar con un contendor imperial y sin tapujos. Por supuesto, también se logró permear algo de más calado: la cultura política y la popularización de la civilisation française que tan buenos retornos le ha dado. 


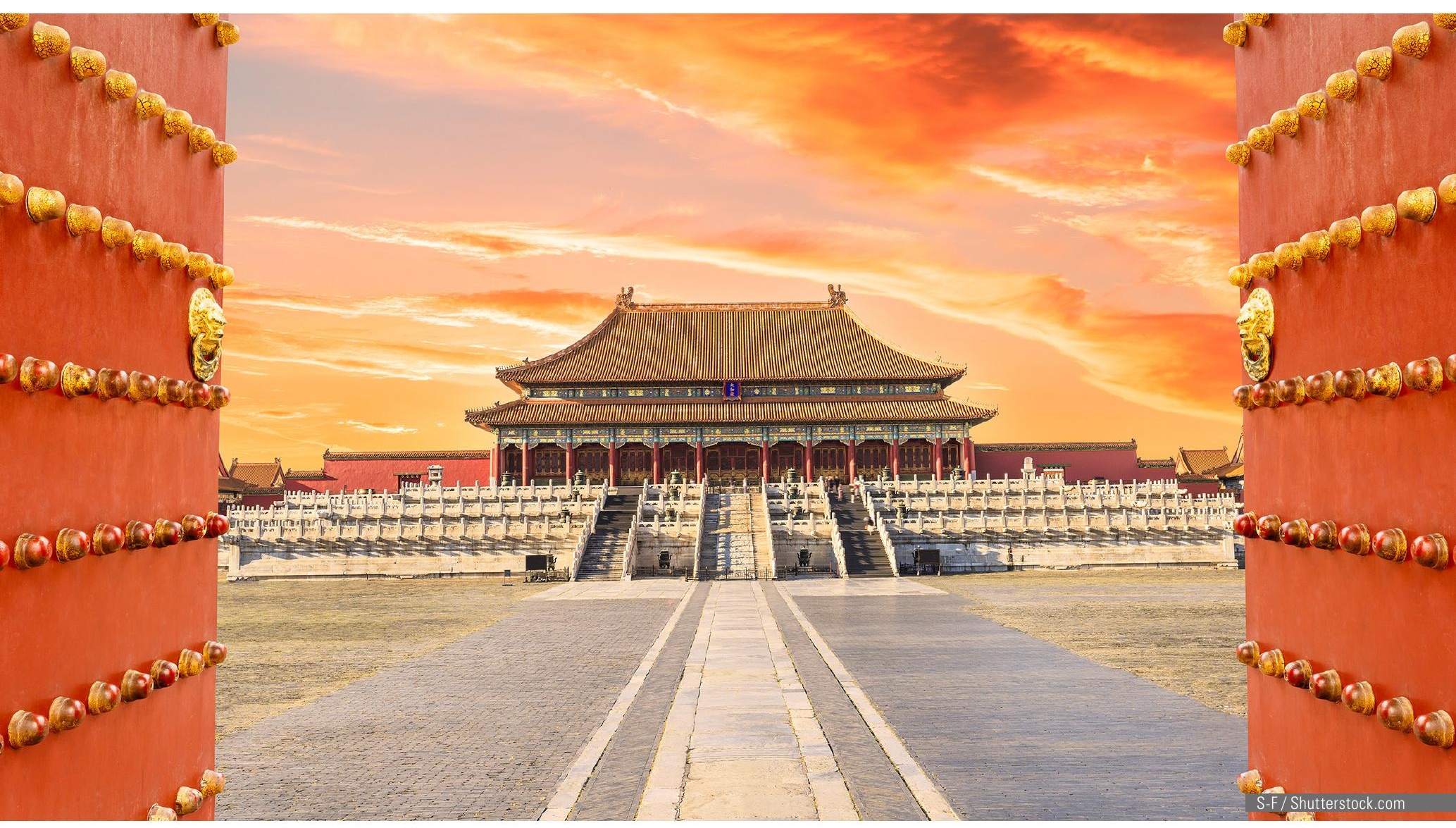

Después de la independencia, o mejor, desde el proceso que condujo a la independencia, la influencia francesa en estas tierras de América fue notable. Y su reinado se extendió hasta los años que siguieron al final de la segunda guerra mundial cuando los Estados Unidos incursionaron con su presencia dinámica.

Mi generación experimentó el coletazo del cambio que tuvo su punto de inflexión en los 60 cuando el francés, antes indispensable, al igual que lo europeo, debió cederle el lugar al inglés en la enseñanza secundaria. Y de igual manera, los mercados tuvieron que doblegarse ante el "made in USA". Pero la inercia era inevitable. Aún recuerdo la voz del poeta mismo, la de José Umaña Bernal, en la emisora HJCK El mundo en Bogotá:

\footnotetext{
Cuando yo digo Francia, es como si dijera

dulzura, y fortaleza, y amor, y gloria, y gracia;

como si el mundo hubiera nacido solamente

para escuchar un nombre: cuando yo digo Francia.

(Umaña, 1975).
}

Tales eran los aires que se respiraban y que explican por qué la llegada de lo "americano" no fue bien recibida. Los viejos rezongaban al ver que la elegancia y el buen gusto eran dejados al garete y eran sustituidos por la rudeza y la simplicidad del cowboy. Atrás quedaba el esprit de finesse del que hablaba Pascal.

Tanto fervor por las fuentes originarias que proveen de una guía a un pueblo no es excepcional. Hoy todavía mantenemos como referente el pensamiento que nos legaron los griegos. Y al igual que nosotros, los japoneses dirán lo mismo de lo que recibieron de la China. Por supuesto, cambia el grado de aceptación, de influencia. Se pasa de momentos, de ciclos de esplendor a otros de ensombrecimiento. La diferencia es que en la actualidad mientras el aporte de occidente reclama la universalidad, los chinos, más modestos, beben de sus propias raíces y de los aportes extranjeros que han asimilado 
como el budismo o el marxismo. De igual forma, nuestro perfil se inclina más hacia lo global mientras que el de ellos se inclina más hacia la órbita de su nacionalismo. Lo cual no significa encerramiento. Probablemente muchas cosas emprenderán, o ya emprendieron el viaje hacia fuera como lo hiciera en el pasado la exportación de sus conocimientos y de sus tecnologías, según lo expuso Joseph Needham en su extraordinaria obra Science and Civilisation in China (Needham, 1954-1986).

Hasta dónde pueda llegar el impulso, está por verse. No debe perderse de vista que hace medio siglo se leía con asombro el libro de Servan-Schreiber, El desafío americano (1967). Y unos años más tarde, en 1970, la obra de Kahn y Pepper, El desafío japonés (1979) que generaba mayores inquietudes por tratarse de algo más desconocido. En el primer texto se exponían los retos que le representaban a Europa la llegada, por no decir la invasión, de capitales estadounidenses al continente que pronosticaban un deterioro de lo europeo. En el segundo se hablaba de manera alarmante sobre lo que significaría para occidente el milagro japonés que se había hecho visible en 1964 con los juegos olímpicos de Tokio y la inauguración del tren bala, el Shinkansen. Ninguno de los dos libros acertó cabalmente, pero las expectativas que se nos presentan hoy en día no son tan distantes a las que nos hicieron reaccionar hace medio siglo.

Lo que puede rescatarse de la historia es que los niveles de absorción de lo que nos llega de afuera tiene sus tiempos y sus grados. Nadie podría negar los espacios que han creado en Europa las formas de pensar de los Estados Unidos y sus gustos así no hayan perforado irremediablemente lo que se conoce como los valores europeos. Y lo mismo puede decirse de los japoneses en el mundo occidental que ha sido invadido por las barras de sushi y por el interés en el zen. La gran diferencia en el caso de estos dos ejemplos es que, los estadounidenses han desarrollado una ideología que no solamente identifica su influencia, sino que la delimita y la fortalece. Japón, por su parte, no ha logrado hacer lo mismo. Su apuesta habría que buscarla más en el campo de la estética. Ahora, en el caso chino es evidente el hondo calado de su pensamiento, acumulado en el curso de la historia y que ha modelado nuevos y propios intentos para entenderse y guiarse en su desarrollo y que en la formulación de lo que se conoce como los valores chinos puede abrirse campo a nivel internacional. Pero, al igual que en el pasado, lo notable es que las distancias no se borran.

En el texto Orientem versus de 1938 (Valéry, 1988), el poeta nos advertía que "ante la perplejidad de tantos nombres e imágenes, quien se oriente hacia el este, será incapaz de lograr una figura clara o un pensamiento definido". Después de ocho décadas es poco lo que ha cambiado la situación. Seguimos perplejos e inhábiles para descifrar con certeza lo que ocurre por esas latitudes. Y gran parte de ese desconcierto hay que buscarlo en las dificultades que surgen cuando llamamos como igual a lo distinto, o entendemos como lo mismo algo que es diferente. Por ello, para avanzar, requerimos de pasos cuidadosos y mesurados que nos cuiden de distracciones y nos procuren concentración.

En 1978, la República Popular de China (RPC) inició un proceso de modernización cuyos resultados la han colocado en el grupo de países que lideran el desarrollo mundial. Hace cuatro décadas el pulso de lo que ocurría por aquellos lados era débil y las noticas que nos llegaban no permitían formarse una idea clara de lo que estaba sucediendo. Los pasos que se daban bajo la etiqueta de socialismo de mercado conducían a pensar que un giro del gobierno estaba llevando al país hacia el capitalismo. Manera un poco simplista con la que se quiso asimilar el proceso en esas orillas y poca credibilidad se le dio a los cuatro principios sobre los que Den Xiaoping cimentó su proyecto. 
Parecían contradictorios y no generaban confianza. Pero con el tiempo serían la clave para entender lo que sucedió. Tales pilares consistían en mantener la vía socialista, la dictadura del proletariado, el liderazgo del Partido Comunista Chino (PCCH) y la ideología marxista-leninista unida al pensamiento de Mao. Si bien la introducción del mecanismo de mercado en estos últimos cuarenta años podría interpretarse como un cambio hacia el capitalismo, lo cierto es que China continúa siendo un régimen comunista en el que las empresas estatales juegan un papel preponderante mientras las privadas se ciñen a las guías gubernamentales.

En el $19^{\circ}$ Congreso del partido comunista de China, celebrado en octubre de 2017, se ha mostrado no solo una consolidación del sistema, sino que lo ha llenado de confianza frente a los retos que impone el mundo actual. Luego de varios años bajo la influencia de Deng que estableció como directriz la de no conducir a China al protagonismo internacional, en este último Congreso se hizo evidente y sin tapujos que los tiempos han cambiado.

Para entender estos episodios seguramente sirvan dos ejemplos. El primero, que está relacionado con el sentido del tiempo en la cultura china, es el caso de Hong Kong. Como se recuerda, en los primeros años de la década de los 50, Inglaterra le ofreció la devolución a Zhou Enlai quien dijo que, por el momento, no estaban interesados y esperaron casi medio siglo para llevarlo a cabo. Y el segundo, que obedece a causas diferentes, fue el giro de la política exterior china hacia una participación más activa, después de que las fuerzas de la OTAN lideradas por los Estados Unidos bombardearan su embajada en Belgrado, en mayo de 1999. Estamos así ante dos fuerzas determinantes de diverso origen. Las internas que tienen su tempo y las externas que no se esquivan. Lo que estamos viviendo es el resurgir poderoso de una nación que desde la guerra del opio fue arrodillada y su resurgimiento en medio de una crisis innegable del liberalismo occidental.

Por eso, al concluir el Congreso $19^{\circ}$, no debe verse como extraño el que la prensa china se pronunciara así:

A finales de 2016 había 13 países cuya población excedía 100 millones. Diez de ellos son países en desarrollo. El éxito de China prueba que el socialismo puede prevalecer yserelcaminoparaqueotros paísesendesarrolloemulenyalcancenlamodernización. China es ya lo suficientemente fuerte, determinada y capaz de contribuir más a la humanidad. El nuevo orden mundial no puede estar dominado solo por el capitalismo y occidente y el tiempo para el cambio llegará (China Daily, 10,24,2017).

La manera como la cultura de cada pueblo se va tejiendo no es perceptible, se elabora sutilmente. Como diría Oscar Wilde, de manera elegante, es decir, sin que nadie se dé cuenta. Con hilos y colores algunas veces más o menos gruesos y resistentes y otras con matices más vistosos o más diluidos, va surgiendo la trama. En todos los casos, y en particular en el de China, no podemos aventurar qué tan hondo habrá de llegarnos su influencia. Pero sí es previsible que suceda y eso debería ser suficiente para estimular nuestro interés en conocerla y en promover la interlocución con esta nueva aventura humana.

Lo que observamos hoy día es un proyecto de gran aliento que se enmarca en lo que se ha denominado "el sueño chino" (chinese dream) y que se quiere hacer realidad sobre el eje que se conoce como la "vía china" (chinese road) que se inspira en el socialismo con características chinas. 


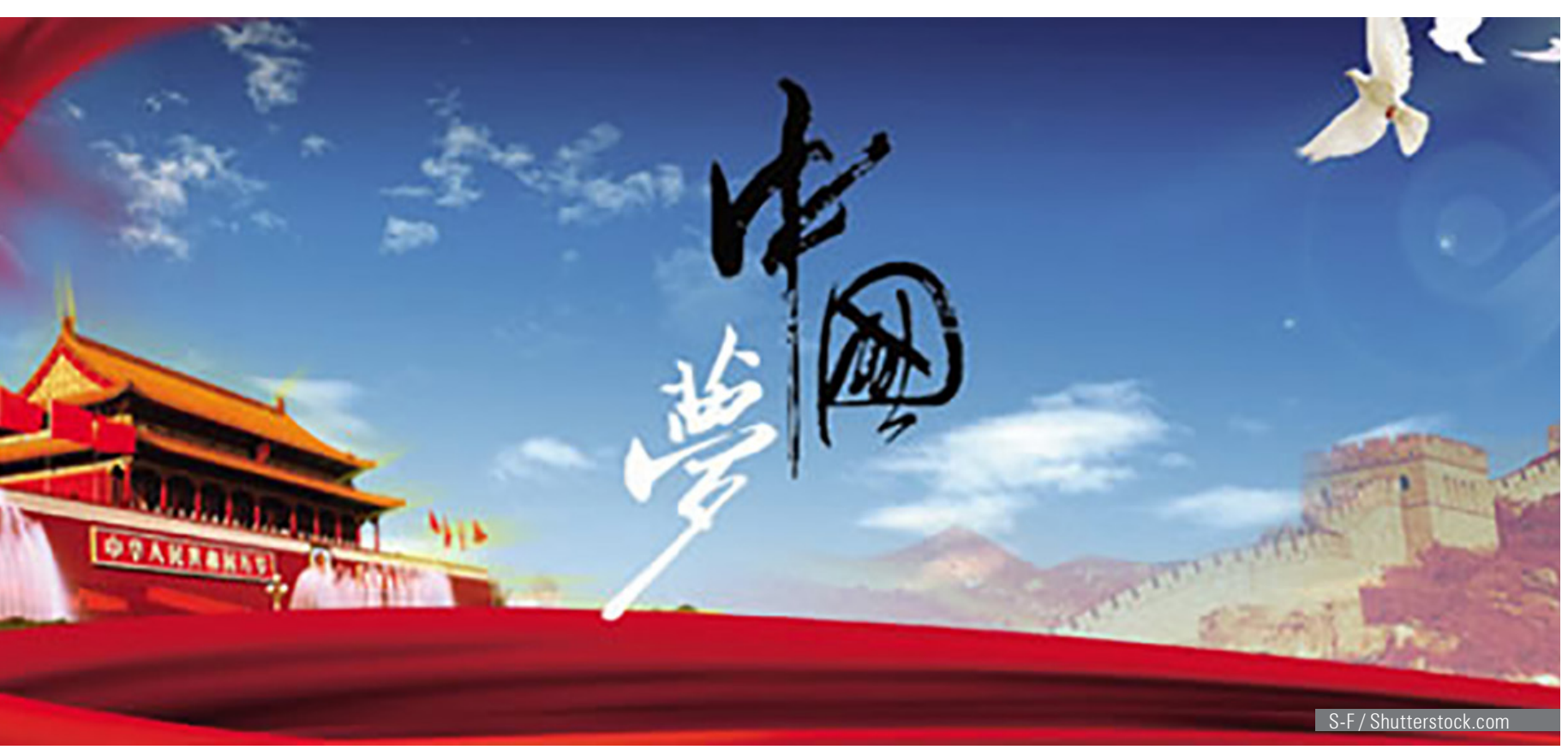

\section{El sueño chino}

Uno de los textos más conocidos del repertorio daoísta es el sueño de la mariposa de Zhuang Zi. Allí se cuenta que Zhuang Zhou soñó que era una mariposa, pero al despertar no lograba discernir si él era una mariposa o la mariposa era Zhuang Zhou (Zhuang Zi, 1998). El novelista Xiaolu Guo se inspiró en este viejo poema para estampar al inicio de su manifiesto el siguiente sueño:

Tuve un sueño. Soñé ser una gran nación. Yo era el Estado. Mi poder se extendía sobre la tierra y sus gentes. Sentí que el poder fluía por entre mis venas y lo sentí fuerte en mi corazón. Ninguna parte de mi cuerpo podía desobedecerme sin ser castigado. Yo era China. Entonces desperté. Como Zhuang Zi que soñaba ser una mariposa, yo me preguntaba: ¿era un hombre que se despertaba de un sueño en el que era China, o era la China despierta el hombre que dormía? (Guo, 2016).

La mención de Zhuang Zi deja claro hasta dónde quiere remontarse el escritor. Y de la misma manera, la alusión al Dr. Martin Luther King Jr. no es gratuita: no se puede esquivar la realidad que no es estática y que se mueve en permanente tensión frente a las contradicciones naturales que conllevan los avances de todas las sociedades.

Desde tiempos inmemoriales, los sueños han sido objeto de interés de todos los pueblos. Han sido medios para descifrar el futuro como también para guiarlo. Lo que vemos en la actualidad es que los chinos sueñan otra vez. Hoy es el sueño que propone Xi Jinping pero que no es novedad. Ya lo había acariciado Deng Xiaoping y antes de él Mao en 1956. Es el sueño cuyo objetivo es recuperar las pasadas glorias de China que son inmemoriales. Aunque podría uno remontarse hasta el emperador Amarillo bastaría con acudir a Marco Polo cuando se refería a Kublai Kan:

...tanto en número de súbditos como en extensión de territorio o monto de sus rentas, sobrepasa en mucho a todos los soberanos que hayan existido o que existan sobre la faz del mundo y no ha sido ninguno de ellos respetado y acatado con tanta obediencia por aquellos a quienes gobiernan (Polo, 1976). 
Y no se puede olvidar que durante 18 de los pasados 20 siglos el PIB chino ha sido superior al de cualquiera otra nación. Sin ir muy lejos, en 1820, cuando la dinastía Qing venía en caída, su economía alcanzaba el 30\% del PIB global (Bregolat, 2015). Sin embargo, no todo se reduce a lo material. Deben agregarse, sin duda, los legados culturales que han florecido en su territorio durante milenios.

Con la publicación de La Interpretación de los sueños de Sigmund Freud en 1899, los sueños fueron llevados al campo de la sicología. Pero su influencia en la vida humana se extiende hacia más variados horizontes.

Los sueños son una parte esencial de la experiencia humana y pueden ser interpretados de varias maneras, por ejemplo, como fuente de poder para alcanzar un nivel más profundo de comprensión. Los sueños han sido un objeto constante de interés desde los tiempos más remotos hasta el presente (...) los sueños han jugado un papel poderoso en la historia. Los historiadores han reportado sueños de líderes famosos que han cambiado el curso de los acontecimientos como una forma de análisis cultural. Burke ha sugerido que, si "Ios sueños tienen una capa cultural de significado, así como una capa personal y universal, se les abren posibilidades aún más estimulantes a los historiadores" (Ho, 2018).

Por supuesto, la lista de sus efectos no se detiene en la historia y debe ampliarse para incluir el arte, la religión, la sociología, la antropología, la filosofía, la teología y la literatura. Pero en el caso chino, especialmente cuando buscamos la raíz del sueño nos encontramos con que:

Entre las civilizaciones de la era axial ${ }^{1}$, se dice que la antigua China es la única que "ha tenido el sentido de mirar hacia atrás a partir de la disrupción actual hasta un imperio y una cultura que floreció en el inmediato pasado con el fin de encontrar soluciones a los problemas contemporáneos. Una profunda conciencia histórica llevó a los viejos filósofos chinos a mirar atrás hacia su perdida "edad de oro", particularmente la de Xhou, al construir una nación ideal desde lo más hondo de la historia china (Ho, 2018).

Y en este sentido, se nos revela una característica novedosa para el análisis de estos desarrollos. Mirar atrás no solamente sirve para estimular sino para iluminar y formular soluciones para hoy y mañana.

Aquí resulta útil acudir a la comparación entre el "sueño americano" y el "sueño chino" que elabora J. Kai (2017). Lo primero que debe recordarse es que la propuesta del sueño americano provino de la época de la depresión. Adams (1931) lo definió en su libro y en uno de sus artículos de prensa lo precisó en estos términos:

El sueño es una visión de una vida mejor, más profunda, más rica para todos los individuos sin importar la posición que él o ella ocupen en la sociedad en razón a las circunstancias de su nacimiento. Ha sido un sueño la oportunidad de ascender en la escala económica pero tanto o más que una oportunidad para desarrollar nuestras capacidades al máximo sin restricciones injustas de casta o de costumbre. Con esto se ha ido la esperanza de mejorar las condiciones físicas del vivir, de disminuir las fatigas y las ansiedades de la vida diaria (Adams, 1933).

Como puede observarse, lo que se persigue es un objetivo material económico- que le ofrezca iguales condiciones al individuo independientemente de sus condiciones. No se trata de una meta colectiva. Y esto marca una gran diferencia con lo que busca China. Siguiendo al citado Kai (2014) en The Diplomat, mientras el sueño americano puede expresarse como "mi sueño", el chino se representa como "nuestro sueño", lo cual tiene unas raíces profundas en una cultura preponderantemente colectivista.

1 Sobre la era axial puede consultarse Arnason, Eisenstadt y Wittrock, 2005. 
Lo que buscan los chinos no es nuevo. Desde finales del siglo XIX se ha incubado el deseo de superar las épocas del colonialismo occidental que tanto los golpeó y lograr lo que han llamado y siguen llamando el rejuvenecimiento de China. En términos económicos, los siguientes datos son elocuentes:

Tabla 1. PIB, 1500 - 2003 (millones de USD de 1990)

\begin{tabular}{|l|c|c|c|c|c|}
\hline \multicolumn{1}{|c|}{ Años } & 1500 & 1700 & 1820 & 1950 & \multicolumn{1}{c|}{2003} \\
\hline China & 61,8 & 82,8 & 228,6 & 244,985 & $6,187,984$ \\
\hline Asia del Este & 150,822 & 217,38 & 397,207 & 885,111 & $15,081,356$ \\
\hline Europa Occ. & 44,183 & 81,213 & 159,851 & $1,396,078$ & $7,857,394$ \\
\hline E.E. U.U. & 800 & 527 & 12,548 & $1,455,916$ & $8,430,762$ \\
\hline
\end{tabular}

Fuente: elaboración propia, información basada en Maddison (2007).

De representar el 30\% del PIB mundial en 1820, se pasó al 5\% en 1950. Al mismo tiempo, Europa y Estados Unidos subieron de un poco menos del $25 \%$ al $50 \%$ en el mismo período. El rejuvenecimiento que se ha hecho visible en las últimas décadas es notorio y sin duda ha afectado el ánimo de los chinos cuando el sueño comienza a volverse realidad. Por supuesto no puede dejarse de lado la posibilidad de una pesadilla. Pero tanto la probabilidad de éxito como de tropiezos estimula el interés de occidente para hacerle seguimiento al proceso y para tratar de descifrarlo.

Sobre cómo ha evolucionado en las últimas décadas el tema, el profesor Angang Hu de la Universidad de Tsinghua en Beijing hace una síntesis muy descriptiva en el libro que acaba de publicar (Hu, 2018). Este texto escrito en 2012 marca las siguientes etapas históricas que llegan hasta el $18^{\circ}$ Congreso del partido cuando se eligió a Hu Jintao:

- Mao Zedong, el fundador del concepto de la vía china.

- Deng Xiaoping, el creador.

- Jiang Zemin, el defensor y continuador.

- Hu Jintao, el seguidor y re-creador (Hu, 2018).

\section{La inspiración de Mao}

A lo largo de su vida, Mao expresó su ideal de futuro para su país y su gente. Uno de los textos que posiblemente marcan con más precisión sus metas es el discurso Sobre el Centralismo Democrático que pronunció el 30 de enero de 1962 ante una conferencia ampliada de trabajo convocada por el Comité Central del PCCh en el que expresó:

Construir una economía capitalista grande y poderosa tomó de 300 a 400 años; ¿qué habría de malo en construir una economía socialista grande y poderosa en nuestro país en los próximos cincuenta o cien años? Los próximos cincuenta o cien años serán un período épico de cambios fundamentales en el sistema social del mundo, un período de grandes consecuencias que no se puede comparar con ninguna otra época del pasado. Viviendo en tal período debemos estar preparados para librar grandes batallas de manera diferente, en muchos aspectos, a las formas de lucha de los períodos anteriores. A fin de llevar a cabo esta tarea debemos luchar por combinar la verdad universal del marxismo-leninismo con la realidad concreta de la construcción socialista de China y con la realidad concreta de la futura revolución mundial, y mediante la práctica llegar a comprender gradualmente las leyes objetivas de la lucha (Mao, 1962).

Tal como se observa, se proponía un proceso de largo aliento que tendría una fase interna que, en su momento, podría extenderse al resto del 
mundo. De igual forma se planteaba como guía de esta empresa, la ideología marxista-leninista, pero adaptada y armonizada con el pensamiento y las realidades particulares de China. Con esto se hacía hincapié en la necesidad de construir sobre la base del pragmatismo y no meramente de las ideas.

Volver los ojos a lo autóctono también respondía a la necesidad de adaptarse al vacío que dejó la cooperación rusa cuando se deterioraron las relaciones con Moscú. Es necesario mencionar de igual manera lo que fueron los años que siguieron después de este rompimiento hasta la muerte de Mao en 1976. Bastaría con recordar la Revolución Cultural y todo lo que significó en términos humanos. No obstante, nadie se atreve a pasar por encima del período maoísta sin el cual no es posible entender lo que sucedió después incluidos tanto éxitos como fracasos

\section{El sueño de Deng Xiaoping}

Tras la muerte de Mao y un corto período de transición, el poder pasó a manos de Deng Xiaoping quien, en 1978, emprendió las reformas de apertura y modernización que cambiaron el rumbo del desarrollo de China. El país se había transformado, pero estaba maltratado y pobre. Deng partió de reconocer una realidad muy simple: si no se crea riqueza no se puede repartir nada. Ese es el sentido de su lema "enriquecerse es glorioso". Y desde una perspectiva pragmática también entendió que el enriquecimiento sería inequitativo, que unos ganarían más pero que con el tiempo se podría llegar a un equilibrio. El asunto era de tiempo, como lo había señalado Mao. Con Deng, el gran sueño de poder de Mao (the great power's dream) que en el 56 había propuesto como meta la de alcanzar a los Estados Unidos en 2006, comenzó a perfilarse con más precisión.

Deng logró reformular el sistema socialista llevándolo hacia lo que se ha llamado un socialismo con características chinas, o también socialismo de mercado, que introduce mecanismos capitalistas sin desvirtuar el marxismoleninismo-maoísmo como fuentes doctrinales. Y propuso como objetivos el de alcanzar una sociedad de clase media para el 2030 y superar a los Estados Unidos en 2050. La propuesta del socialismo con características chinas ha tenido un efecto político muy interesante que hace más comprensible sus alcances. Si bien en todas las sociedades surgen fuerzas y opiniones encontradas, tras el descalabro que provocaron las políticas de Mao, el país tuvo que enfrentar un debate polarizado. Algunos pedían defender a ultranza la ideología que se había instalado y que se entendía como el paso correcto. Los opositores, que eran vistos como el demonio, se iban al otro extremo que propiciaba un giro hacia el capitalismo. De esta confrontación entre el oro y la escoria, surgió la solución de Deng que esquemáticamente puede describirse como la del justo medio o, como dirían otros, de la tercera vía.

\section{El sueño de Jiang Zemin}

Jiang, quien sucedió a Deng cuando este se retiró en 1989, supo mantener el proyecto y además enriquecerlo con su propuesta de las tres representaciones incorporadas luego a la Constitución y que reconocieron a los empresarios privados como actores económicos. También a su gobierno se debe el ingreso de China a la OMC. Aunque se debate entre los especialistas sobre la perdurabilidad de su gestión, lo cierto es que este tipo de medidas aclimataron el terreno para avanzar hacia un desarrollo más complejo. 


\section{El sueño de Hu Jintao}

Hu, el primer líder que no participó activamente en la revolución, llegó al poder en 2004. Su aporte con mayores proyecciones es lo que se ha llamado la doctrina del desarrollo científico que propende por la construcción de una sociedad armoniosa en un proceso gradual.

El mandato de Hu Jintao terminó en 2012 cuando Xi Jinping fue elegido en su reemplazo. En el informe de $\mathrm{Hu}$ al $18^{\circ}$ Congreso del partido hizo una presentación de las políticas durante su período en relación con las estrategias para mantener e impulsar el sistema. De manera sintética, el reconocido observador y analista Xulio Ríos lo expresa así:

Hu Jintao ha reivindicado las ideas-fuerza principales que han impregnado su mandato. De una parte, la concepción científica del desarrollo. De otra, la justicia social. En el primer caso, cabe significar el "triunfo" de Hu Jintao al lograr inscribir su noción del desarrollo científico en el frontispicio teórico del PCCh. Es la primera vez que esto ocurre y con seguridad se llevará a los Estatutos del PCCh a lo largo de este congreso. También tiene repercusiones a otros niveles. Por ejemplo, hasta ahora los ejes centrales de la política oficial se referían a la construcción económica, social, cultural y política. No obstante, ahora emerge un quinto elemento, el medio ambiente, reflejando la creciente importancia de la ecología en las preocupaciones oficiales (Ríos, 2012).

\section{El sueño de Xi Jinping}

Desde cuando llegó al poder, Xi ha fortalecido el tema de manera señalada y luego de su reelección en el 19 Congreso celebrado en octubre de 2017 ha hecho más evidentes las aspiraciones de su gobierno y de su pueblo, tal como se planteó al comienzo de este escrito. En esta nueva etapa, la más ambiciosa hasta ahora, la vía china, es decir la modernización socialista con características chinas, toma una nueva dirección. Los éxitos económicos, los avances militares y la gradual participación en los asuntos del mundo contemporáneo, han hecho aparecer el modelo como un sistema exportable. A ello contribuyen, sin duda, las crisis del liberalismo en occidente y la incertidumbre que generan los movimientos políticos y sociales en Europa, incluido el Brexit, tanto como los giros en la política internacional de los Estados Unidos en el gobierno del presidente Trump.

Kevin Rudd, ex primer ministro laborista de Australia quien se mantiene muy activo internacionalmente, ha comentado lo que sigue sobre Xi y su gobierno:

Su visión central y animadora se centra en su concepto del "Sueño de China" (zhongguomeng 中国 梦) que a su vez tiene dos objetivos: lograr una "China moderadamente acomodada" (xiaokang shehui 小康 社会) para el año 2021 cuando el Partido celebre su centenario; y "una rico y poderosa" (fuqiang 富强) China para 2049 en el centenario de la República Popular. Realizar el Sueño de China, según $\mathrm{Xi}$, requiere una segunda fase de reforma económica transformadora. Él no ve ninguna contradicción en el enjuiciamiento de reformas de mercado más profundas para lograr sus objetivos nacionales, mientras implementa nuevas restricciones en la libertad política individual. De hecho, él ve esto como la esencia del "Modelo de China" (zhongguo moshi 中国 in) en contraste con el capitalismo democrático liberal de Occidente, que él describe como totalmente inadecuado para China (Rudd, 2015).

Rudd toca un punto sensible cuando se refiere a las libertades individuales. Este tema, como tantos otros, plantea las profundas diferencias de valores que se cruzan entre China y occidente. Cuál sistema de valores puede calificarse como mejor, es una pregunta que cae bien en el campo especulativo pero que no aporta mucho para entendernos dentro de la diversidad. Veamos la siguiente cita: 
Estrictamente hablando, las ideas tradicionales chinas sobre el valor no equilibran bien la libertad personal y la armonía social y la justicia. O la libertad personal se sacrifica a causa de los llamados intereses grupales, lo que resulta en una autocracia y uniformidad absoluta; o el honor del grupo se ve perjudicado por algún tipo de interés privado, lo que lleva al estado de desunión y de presunción extrema. La ética china sostiene que, para deshacerse de tal dilema ético, la mejor opción no es el castigo por ley sino el estímulo de la simpatía y el sentido de la vergüenza en cada corazón. Esto es lo que dice el Sr. Liang Shuming: "El orden social en China se basa en la etiqueta y las costumbres, mientras que el oeste lo hace en la ley. Para aquellos que dependen de la ley, los derechos y las obligaciones deben ser aclarados sin perturbación mutua; para aquellos que dependen de la etiqueta y las costumbres, lo que necesitan hacer es solo enfatizar tradiciones y costumbres. Si la costumbre y la tradición se mantienen bien, las personas se llevarán bien entre sí. Para juzgar si la relación humana es armoniosa o no, lo más importante es examinar la relación entre un padre y un hijo y entre otros parientes. Es por esta razón que la piedad filial y la honestidad se enfatizan mucho cuando se contratan empleados públicos. Como se menciona en el Libro de la Piedad Filial, la moral, originada y desarrollada a partir de la piedad filial, es la base de la etiqueta y las costumbres (Dai y Li, 2013).

Lo que quiere resaltarse con esta reflexión, es la necesidad de dilucidar el origen de las diferencias para poder acercarnos a ellas, entenderlas, y tender puentes que faciliten el fortalecimiento del respeto hacia los demás. Y la respuesta china, que viene desde los tiempos de Confucio, se llama armonía.

\section{La armonía: la guía}
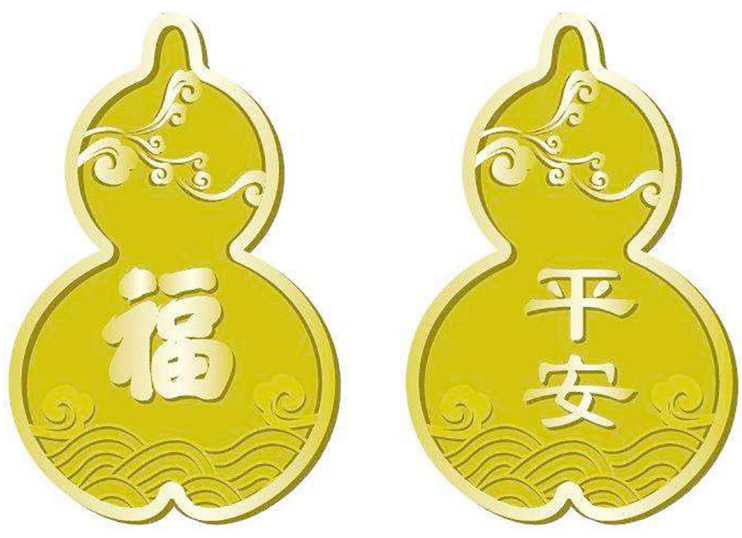

Fuente: http://gansujingshi.com/zoujinjingshi/qiyerongyu/

Liu Mingfu en The Atlantic del 4 de junio de 2014, escribió lo siguiente:

El político francés Georges Clémençeau dijo: "La guerra es demasiado importante como para dejarla en manos de los generales". Charles de Gaulle dijo: "La política es demasiado importante como para dejarla en manos de los políticos". Un experto chino dijo: "El mundo es demasiado importante para ser dejado a América. China necesita 'diseñar el mundo'. China debe liderar el mundo hacia un futuro mejor ". Como diseñadora, China necesita producir un mejor modelo para el mundo que Estados Unidos. Como líder mundial, China necesita producir una mejor agenda política que la estadounidense. China ha dicho que quiere construir un mundo armonioso, y esto es lo que ofrece el diseño de China para el mundo (Liu, 2014). 
La armonía como ideal está anclada en lo más profundo del pensamiento y de las tradiciones chinas. $Y$ al hablar de ideal lo que se quiere señalar es que guía los propósitos de la sociedad así no siempre alcance sus objetivos. Algo como lo que sucede con la ley en sociedades como la nuestra. Ahora bien, cuando se trata de dilucidar qué se entiende en China por armonía, surgen varios escollos pues los fundamentos sobre los que construimos el concepto aquí y allá no coinciden. Y más aún, además de las contradicciones que se presentan existe otra característica del pensamiento chino que dificulta su comprensión: la ambigüedad.

Una sociedad socialista armoniosa es una sociedad que está llena de vitalidad y goza de unidad y concordia. Debemos hacer todo lo posible para aumentar la vitalidad de la sociedad, promover la armonía en las relaciones entre partidos políticos, grupos étnicos, religiones, estratos sociales y compatriotas en el país y en el extranjero, y consolidar la gran unidad de la gente de todas nuestras nacionalidades y todos los hijos e hijas de la China en todo el mundo. ${ }^{2}$

Debemos respetar y comprender plenamente las diferencias de las nacionalidades en sus tradiciones, su idioma, su cultura, sus costumbres y su identidad psicológica que surgen a través de su desarrollo histórico. No podemos ignorar su existencia o cambiarlos por la fuerza. Necesitamos crear activamente las condiciones para disminuir y eliminar las disparidades en el nivel de desarrollo de las nacionalidades (Hao, 2016). ${ }^{3}$

Estos dos textos transcritos por Hao nos remiten a la propuesta de $\mathrm{Hu}$ Jintao de establecer la armonía como guía para avanzar tanto en lo interno como en lo externo. La sociedad armoniosa que se busca no se limita a China. También debe servir de fundamento para la construcción de un mundo en el que quepan todos. Y ello significa reconocer la diversidad como capital principal del humanismo que se quiere construir. El mundo es una suma de culturas, razas, ideologías, religiones, artes, sensibilidades, gustos, etc.

La razón por la cual la armonía puede contribuir a abrir los canales del entendimiento universal, resultan más reconocibles si recurrimos a la etimología de la palabra en chino:

Según el estudio realizado por el erudito chino Wang Guowei (1877-1927), 盉 [he] originalmente significa el utensilio utilizado para mezclar vino con agua a fin de ajustar la densidad del vino. Esto es muy consistente con la definición en Shuo Wen Jie Zi, que define 盉. [he] como verbo: mezclar sabores (tiao wei ye), ya que es común en el idioma chino antiguo convertir un sustantivo en un verbo que indica la función del objeto que el nombre denota. También es natural extender su significado desde el proceso de mezclar sabores hasta el proceso general de mezclar otras cosas también (Li, 2018).

Valga la pena insistir de nuevo sobre las diferencias entre oriente y occidente. Aquí también hacemos uso de las mezclas y entendemos que la buena cocina es la que logra matizar sabores y texturas. Sin embargo, al remitirnos a la primera receta del libro de cocina más antiguo entre nosotros, escrito posiblemente entre los siglos I y III, pero sólo publicado en el XV en Venecia con los títulos de Ars magirica, o Apicius culinarius, o también De re coquinaria libri decem (Apicio, 1987), encontramos la fórmula para preparar el conditum paradoxum o vino aromático. Dice que se usará la espátula para revolver los contenidos. Y descubrimos entonces que entre nosotros no es común derivar un verbo de un sustantivo razón por la cual no contamos con "espatulear". Lo cual nos conduce a dirigir la mirada al pasado para fundar la armonía de occidente en la música, en las matemáticas o en el juego de las esferas celestiales. Pero no en lo terrenal.

2 Decisión del Comité Central del Partido Comunista de China sobre Cuestiones Importantes relacionadas con la Construcción de una Sociedad Socialista Armoniosa.

3 Hu Jintao, "Informe en la Conferencia central sobre trabajo de nacionalidad." 
La mezcla de ilusiones, planes, esperanzas y temores que surgen de la Relación entre China y América Latina] es tan poderosa en su impacto en [América Latina] como lo son los negocios y eventos en sí mismos. . el mayor impacto de China vendrá de lo que lleve a la región a soñar, y lo que América Latina encuentre cuando despierte (Ellis, 2008).

Y agrega lo siguiente:

Esta declaración, parte de una onda reciente pero creciente de académicos, medios de comunicación, gobierno y empresas interés en las florecientes relaciones económicas y políticas de China con las regiones del mundo en desarrollo de América Latina al África y al sudeste de Asia, capta una idea importante, pero a menudo infravalorada. Específicamente, las percepciones y expectativas de los líderes gubernamentales y empresariales, así como los ciudadanos del común en los países de América Latina jugarán un papel crucial para determinar el desarrollo de las relaciones económicas y políticas entre sus países y China (Ferchen, 2011).

Esto reclama una cuidadosa y ponderada reflexión en la medida en que pone sobre aviso a los países que no están incluidos en el proyecto original, es decir, los que quedan por fuera de Asia, África y Europa. Si bien esto no significa que Latinoamérica sea ignorada por China, lo que es evidente es que el interés de los chinos estará focalizado en la venta de sus productos y en la compra de materias primas de esta región. Visto el futuro desde esta realidad, lo que se identifica es el enorme reto que tendrán los gobiernos y empresarios de Centro y Sur América lo mismo que los del Caribe para aprovechar lo que muy probablemente será el centro de desarrollo de esta primera mitad del siglo XXI. Y si ello no está acompañado por acciones ambiciosas y audaces, se puede repetir el aumento de la brecha de desarrollo que ya se experimentó con el crecimiento del Asia del Este en la segunda mitad del siglo XX.

La meta próxima de China es todavía preponderantemente doméstica: alcanzar una sociedad afluente en el 2030, es decir, una sociedad de clase media. Pero ya está planeado y en sus inicios un proyecto ambicioso para compartir el modelo chino con el resto del mundo. Nos encontramos en medio de un proceso cuyos resultados son inciertos como fueron inciertas las predicciones de Kahn y de Servan-Schereiber a las que nos referimos al inicio. Sin embargo, no puede soslayarse el hecho de que por detrás se cuenta con una motivación enorme. Se trata de construir un mundo mejor en el que todos logremos acomodarnos. Pero sería insensato olvidar que la apuesta se hace desde una cultura en la que lo único que se acepta como verdadero es el cambio. Es por esto que lo que puede esperarse con un grado mayor de certeza, es la continuidad de los cambios en la teoría y en la práctica que seguirán inspirando el recorrido.

\section{Referencias}

- Adams, J.T. (1931). The Epic of America. Little, Brown, and Company: Boston. (1933). "America Faces 1933's Realities". New York Times, January 1, 1933.

- Apicius, M. G. (1987). Gastronomía en la antigua Roma imperial. Editorial Coloquio: Madrid.

- $\quad$ Arnason, J. P., S. N. Eisenstadt y B. Wittrock, Eds. (2005). Axial Civilizations and World History. Brill: Leiden.

- Bregolat, E. (2015). The Second Chinese Revolution. Palgrave Macmillan: Hampshire.

- China Daily $(10,24,2017)$. Milestone congress points to new era for China, the world. Recuperado de http://www.chinadaily.com.cn/china/19thcpcnationalcongress/2017-10/24/content 33648051.htm

- Dai, M y J. Li (2013). "Three Doubts about the Comparison between Western and Chinese Views of Harmony from the Perspective of Axiology". En: Deshun Li, Ed. Values of Our Times Contemporary Axiological Research in China. Springer-Verlag Berlin: Heidelberg.

- Ellis, E. R. (2008). China in Latin America: The What's and Wherefores. Lynne Reinner Publishers: Boulder.

- Ferchen, M. (2011). "China-Latin America Relations: Long-term Boon or Short-term Boom?" En: The Chinese Journal of International Politics, Vol. 4, 2011, 55-86. 
- Guo, X. (2016). "Words from a Brick in the Wall (Or, What the Dialectical Poet Tries to Say but Fails to Say, and What He Might Whistle or Sing)". En: Ariel: A Review of International English Literature, 47: 1-2. pp. 391-395

- Hao, S. (2016). How the Communist Party of China Manages the Issue of Nationality, Springer-Verlag: Berlin Heidelberg?

- Ho, W. C. (2018). Culture, Music Education, and the Chinese Dream in Mainland China. Springer Nature: Singapore.

- Hu, A. (2018). China's Road and China's Dream. Springer Nature: Singapore.

- Kahn, H. y T. Pepper (1981). El desafío japonés. Editorial Norma: Bogotá.

- Kai, J. (2014). "The China Dream Vs. The American Dream". En: The Diplomat, September 20, 2014. Recuperado de https://thediplomat.com/../the-china-dream-vs-the-american-dream/ (2017). Rising China in a Changing World. Palgrave Macmillan: Singapore.

- Li, C. (2008). "The Ideal of Harmony in Ancient Chinese and Greek Philosophy". En: Dao 7:83.

- Liu, M (2014). "The World Is Too Important to Be Left to America". En the Atlantic. Recuperado de

- https://www.theatlantic.com/international/archive/2015/06/china-dream-liu-mingfu-power/394748/

- Maddison, A. (2007). Contours of the World Economy, 1-2030AD. Essays in Macro-Economic History. Oxford University Press: Oxford.

- Mao, Z. (1962). “Sobre el Centralismo Democrático". Recuperado de http://www.acgcr.org/mlm_archivos/ Archivos/mtscde01.htm 7/04/18

- Needham, J. (1954-1986). Science and Civilization in China. 15 vols. Cambridge University Press: Cambridge.

- Neilson, B. (2017) "Belt and Road: State transformation and large technical systems". Recuperado de https:// www.lowyinstitute.org/the-interpreter/belt-and-road-state-transformation-and-large-technical-systems

- Polo, M. (1976). Viajes. Editorial Porrúa: México.

- Ríos, X. (2012). "XVIII Congreso del PCCh: Segunda lectura del informe de Hu Jintao". Recuperado de http://www.asiared.com/client/print/print_notice.php?!DN=3318

- Rudd, K. (2015). U.S.-China 21, the Future of U.S.-China Relations under Xi Jinping. Belfer Center for Science and International Affairs, Harvard Kennedy School: Cambridge.

- Servan-Schereiber, J. J. (1968). El desafío americano. Editora Zig-Zag: Santiago de Chile.

- Shawcross, E. (2018). France, Mexico and Informal Empire in Latin America, 1820-1867. Palgrave Macmillan: London.

- Umaña Bernal, J. (1975). Itinerario de fuga. Editorial Kelly: Bogotá.

- Valéry, P. (1988). En: Regards sur le monde actuel et autres essais, Paris, Gallimard, coll, 1988.

- Zhang, Y. (2014). "The idea of order in ancient Chinese political thought: A Wightian exploration". En: International Affairs, 90, pp. 167-183.

- Zhuang Zi (1998). Los capítulos interiores de Zhuang Zi. Trotta: Madrid 